\title{
Robust Optimization Model with Shared Uncertain Parameters in Multi-Stage Logistics Production and Inventory Process
}

\author{
Lijun Xu ${ }^{1}$, Yijia Zhou ${ }^{2, *}$ and Bo Yu ${ }^{3}$ \\ 1 School of Science, Dalian Maritime University, Dalian 116026, China; lijun_xu@dlmu.edu.cn \\ 2 School of Computer \& Software, Dalian Neusoft University of Information, Dalian 116023, China \\ 3 School of Mathematical Sciences, Dalian University of Technology, Dalian 116024, China; yubo@dlut.edu.cn \\ * Correspondence: zhouyijia@neusoft.edu.cn
}

Received: 24 December 2019; Accepted: 2 February 2020 ; Published: 7 February 2020

\begin{abstract}
In this paper, we focus on a class of robust optimization problems whose objectives and constraints share the same uncertain parameters. The existing approaches separately address the worst cases of each objective and each constraint, and then reformulate the model by their respective dual forms in their worst cases. These approaches may result in that the value of uncertain parameters in the optimal solution may not be the same one as in the worst case of each constraint, since it is highly improbable to reach their worst cases simultaneously. In terms of being too conservative for this kind of robust model, we propose a new robust optimization model with shared uncertain parameters involving only the worst case of objectives. The proposed model is evaluated for the multi-stage logistics production and inventory process problem. The numerical experiment shows that the proposed robust optimization model can give a valid and reasonable decision in practice.
\end{abstract}

Keywords: robust optimization; duality theory; uncertain set; logistics production; inventory process

\section{Introduction}

In real life, we usually encounter some problems with uncertain data. In order to solve those problems properly, we must consider them within an uncertain scope. For problems with uncertainty, there are many approaches to handle them, such as sensitivity analysis, stochastic programming, and robust optimization. In this paper, we focus on one of the popular methodologies, robust optimization (RO), which addresses optimization problems with uncertain parameters described using uncertainty sets other than probability distributions. The aim of robust optimization is to determine a solution that is feasible for any realizations of uncertain parameters, and to be optimal for the worst-case scenario of these uncertain parameters. In other words, robust optimization gives a decision which is ensured to be "good" for all possible realizations of uncertain parameters.

\subsection{Review of Robust Optimization}

In recent decades, robust optimization has been popularized to handle practical problems with incomplete messages, namely, with uncertain data. The primary work by Bertsimas et al. [1] surveys research on robust optimization both theoretically and practically. For linear optimization problems, Brown et al. [2] propose a special robust optimization with constructed uncertainty sets. Ben-Tal et al. [3] relax the standard robustness by varying the protection level across the uncertainty set and extend the framework of robust optimization. There are also some works on robust optimization for the mixed integer stochastic optimization problem. For example, Bertsimas et al. [4] show that there can be a good approximation of the static robust solution for two-stage mixed integer stochastic problem 
under fairly general assumptions. Delage et al. [5] demonstrate that the associated distributionally robust stochastic programming with a large range of objective functions can be solved efficiently by the proposed model with uncertainty in the form of distribution and moments. Luo et al. [6] investigate robust optimization equilibria in game theory with two players by estimating a bounded asymmetric uncertain set. $\mathrm{Xu}$ et al. consider linear regression problems with least-square error in [7]. Later, $\mathrm{Xu}$ et al. [8] connect robust optimization and distributionally robust stochastic programming and show that the solution of the RO problem is also a solution of the latter problem. Distributionally robust optimization is later studied as one popular area of robust optimization including convergence, algorithms, and applications, see [9-13] for examples.

In most of those robust optimization models mentioned above, the same uncertain variable does not exist in either objective or constraints simultaneously. However, in some papers like [14-17], the objective and constraints often contain the same uncertain variable simultaneously. In other words, they share the same uncertain parameters. In this case, researchers usually consider the worst cases over the uncertain variable of the objective and each constraint, respectively. For instance, Tong et al. [17] respectively get the dual forms of the worst objective and the worst constraints under the given uncertainty sets. Recently, Yao [18] also employs this method in the robust multi-stage logistics production and inventory process problem. The resulting optimization of this approach is usually a convex and linear problem that is easy to solve. However, these robust models are overly conservative, since the worst case of each objective and each constraint are handled separately. Therefore, they may result in a highly impossible case-that the same uncertain variable is solved with different values in objective and constraints.

\subsection{Motivation}

Most robust optimization models involve minmax(maxmin) objective functions. The popular approach to handle this kinds of objective is reformulating minmax(maxmin) problems to $\min (\max )$ ones by the dual theorem. For example, the theoretical work in [11] reformulates the inner maximization as a semi-infinite programming through Lagrange dual when solving minimax distributionally robust optimization problems. Another practical work [10] for economic dispatch in energy integrated systems also convert objective with respect to uncertainty to its dual form. Similarly, for max(min) problems (i.e., the worst cases) in constraints, one strategy is to consider its dual forms with respect to uncertain parameters. Like the work in $[17,18]$ separably transforms the worst-case objective and each constraint to the corresponding dual forms under the given uncertainty sets. However, the separable and respective way to convert the worst-case objective and constraints most likely results in the difference value of the uncertain parameter being between the optimal objective and the worst-case constraint. Recently, Zhou et al. [19] propose robust risk-reward optimization models which ensure the same distribution both in the reward and in constraints. Motivated by the above works, we are prompted to focus on a generalized work which only considers the worst case of the objective itself over the shared uncertain variable instead of also considering the worst case of each constraint. We present an innovative model different to the existing robust optimization models which are overly conservative. In this way, the optimal value of the uncertain parameter in proposed model can be the same one in both the objective and constraints. Moreover, it is less conservative naturally and reasonable in practice.

\subsection{Orgnization}

In Section 2, we propose the standard form of the robust optimization model with shared uncertain parameters and compare it with the existing robust model. We will show that the proposed robust optimization model is more reasonable. In Section 3, we utilize the approach to remodel a real robust problem in logistics production and inventory process. Then we reformulate them to tractable forms by dual theory under some assumptions. Numerical experiment conducted in Section 4 shows that the existing robust model is too conservative to have a solution in some cases, but the new robust model is 
validated to forecast earnings and give a good decision to investors. Finally, we conclude this paper in the last section.

\section{Robust Optimization Model with Shared Uncertain Parameters}

The optimization problems with uncertainty are generally formulated as follows:

$$
\begin{array}{cl}
\min _{x} & f(x, \xi) \\
\text { s.t. } & g_{i}(x, \xi) \geq 0, i=1, \cdots, k, \\
& x \in \mathscr{X}
\end{array}
$$

where both the objective and constraints are uncertain with the same random variable $\xi$ and the feasible set $\mathscr{X} \subseteq \mathbb{R}^{n}$. We assume that the variable $\xi$ belongs to a compact uncertainty set $U$. Generally, the model in Equation (1) is hard to solve because of the existence of uncertain parameter. The popular way to handle this problem, like in $[14,16,18,20,21]$, is to consider the worst case of objective and each constraint, respectively. That is,

$$
\begin{array}{ll}
\min _{x} & \max _{\xi \in U} f(x, \xi) \\
\text { s.t. } & \min _{\tilde{\zeta} \in U} g_{i}(x, \xi) \geq 0, i=1, \cdots, k, \\
& x \in \mathscr{X} .
\end{array}
$$

This model is regarded as the classic robust optimization problem.

\subsection{Goal and Method}

The robust optimization model in Equation (2) considers the worst cases of the objective and all constraints with $\xi$, respectively. It is straightforward that the uncertain variable $\xi$ in the respective worst case of the objective and constraints are allowed to be different. This may be an overly conservative assumption since it requires that each constraint should be satisfied for all possible (in particular, the worst case) realizations of the uncertain parameters $\xi$. Therefore, it makes sense to improve these kinds of models, in fact, that one variable should have the same meaning and evaluation at the same scenario. An interesting alternative would be to consider a model in which the optimal uncertain variable in the worst case of the objective itself meets related constraints in Equation (1). Motivated by this consideration, we present the following robust optimization model with shared uncertain parameters (denote as RO_Shared):

$$
\begin{array}{ll}
\min _{x} & \left\{\max _{\tilde{\xi} \in U} f(x, \xi): g_{i}(x, \xi) \geq 0, i=1, \cdots, k\right\} \\
\text { s.t. } & x \in \mathscr{X}_{1},
\end{array}
$$

where the feasible set $\mathscr{X}_{1}=\left\{x \in \mathscr{X}: g_{i}(x, \xi) \geq 0, \xi \in U, i=1, \cdots, k\right\}$. We can easily obtain that model in Equation (3) as it only focuses on the worst case of the objective over $\xi$ which indeed satisfies all constraints simultaneously. This model meets the requirement of the real situation more suitably than the model in Equation (2). We illustrate this point by the following example.

\subsection{Synthetic Examples and Analysis}

Example 1. Compare the following two robust optimization problems:

$$
\begin{array}{cl}
\min _{x \in \mathscr{X}} & \max _{\tilde{\zeta} \in U} 3 x-\xi \\
\text { s.t. } & \min _{\tilde{\zeta} \in U} g(x, \xi)=x-\xi^{2} \geq 0 .
\end{array}
$$




$$
\min _{x \in \mathscr{X}}\left\{\begin{array}{ll}
\max _{\tilde{X} \in U} & 3 x-\xi \\
\text { s.t. } & g(x, \xi)=x-\xi^{2} \geq 0
\end{array}\right\} .
$$

Let $\mathscr{X}=[1,5]$ and $U=[0,2]$. We obtain the optimal solution $x=4$ and $\xi_{1}=0$ in Equation (4), but the constraint reaches its worst case at $\xi_{2}=2$. So Equation (4) is overly conservative since the value of $\xi$ in the optimal solution may not be the same one as in the worst case of the constraint. If we also consider the worst case of the constraint, we may get different situations which would not likely to happen simultaneously in practice. Meanwhile, the optimal solution of Equation (5) is $(x, \xi)=(1,0)$ which ensures intrinsic consistences of $\xi$ in objective and constraints. Clearly, Equation (5) is less conservative than Equation (4) in terms of optimal objective value.

In fact, the model in Equation (2) is a conservative approximation for the new model in Equation (3). We conclude that the model in Equation (2) is feasible $\Rightarrow$ the model in Equation (3) is feasible.

Denote the non-empty set

$$
\mathscr{X}_{2}=\left\{x \in \mathscr{X}: \min _{\xi \in U} g_{i}(x, \xi) \geq 0, i=1, \cdots, k\right\}
$$

as the feasible set of model in Equation (2). According to the definition of the feasible set, for any $x^{*} \in \mathscr{X}_{2}$, we have $\min _{\xi \in U} g_{i}\left(x^{*}, \xi\right) \geq 0, i=1, \ldots, n$. Obviously, we obtain that $g_{i}\left(x^{*}, \xi\right) \geq 0, i=1, \ldots, n$ are satisfied for all $\xi \in U$. Thus $x^{*}$ is the feasible solution of the model in Equation (3).

The above discussion gives rise to the following elementary result.

Theorem 1. The feasible set $\mathscr{X}_{2}$ constitutes a conservative approximation for $\mathscr{X}_{1}$, that is, $\mathscr{X}_{2} \subseteq \mathscr{X}_{1}$.

Conversely, if the model in Equation (3) is feasible, the model in Equation (2) is not always feasible, as demonstrated in the following example.

Example 2. Consider the robust optimization problems in Example 1 with $\mathscr{X}=[1,2]$ and $U=[0,2]$. And then the model in Equation (5) is always feasible for $\forall x \in \mathscr{X}$. Meanwhile, for any $x \in \mathscr{X}, x-4=$ $\min _{\tilde{\xi} \in U} g(x, \xi) \geq 0$ does not hold, hence the model in Equation (2) is infeasible.

\subsection{Real Example in Portfolio Optimization}

Now we take for example the practical portfolio problem. The current robust reward-risk model (see [14-17] for example) is presented as follows:

$$
\begin{array}{cl}
\min _{x \in \mathscr{X}} & \max _{p \in \mathscr{P}} \operatorname{CVaR}_{p}(x) \\
\text { s.t. } & \min _{p \in \mathscr{P}} \mathbb{E}_{p}(x) \geq S_{*},
\end{array}
$$

where $x \in \mathscr{X}$ is the decision variable, $p \in \mathscr{P}$ is an uncertainty set, and $S_{*}$ represents the lowest expected return. Obviously, Equation (6) is a specific example of Equation (2) by separately considering the worst-case distribution in the reward $\mathbb{E}_{p}$ and the risk $\mathrm{CVaR}_{p}$. Intuitively, $\operatorname{argmax}_{p \in \mathscr{P}} \mathrm{CVaR}_{p}(x)$ may most likely be different from $\operatorname{argmin}_{p \in \mathscr{P}} \mathbb{E}_{p}(x)$.

However, the corresponding RO_Shared model in Equation (3) for the robust reward-risk model is

$$
\min _{x \in \mathscr{X}} \max _{p \in \mathscr{P}}\left\{\operatorname{CVaR}_{p}(x): \mathbb{E}_{p}(x) \geq S_{*}\right\} .
$$

It is straightforward to see the difference on the value of $p$ in the optimal solution of the models in Equations (6) and (7). 
It should be clear that the models in Equations (3) and (7) are more flexible than the models in Equations (2) and (6), i.e., it has a larger robust feasible set, enabling a better optimal value while still satisfying all possible realizations of the constraints.

The proposed model in Equation (3) is a generalized model, so we can only provide a framework on how to solve it. That is, the inner maximization problem in Equation (3) can be equivalent to its dual form which is a minimization problem under some assumptions and then the minmax model in Equation (3) can be converted to a minimization problem. Like the specific problems considered in [14-23], we focus on affinely adjustable robust optimization with application to a multi-stage logistics production and inventory process problem.

\section{Multi-Stage Logistics Production and Inventory Process}

For multi-stage (affinely adjustable) robust optimization such as models in [22,23] and so on, the worst cases of both objective and constraints are also considered and converted to their dual forms, respectively. In this way, they may get different optimal values of the same uncertain variable, which is not true in fact. In this section, we remodel the affinely adjustable robust logistics and inventory problem as the new robust optimization in Equation (3).

\subsection{Problem Description}

First, we present the following notations.

T: Set of time intervals $\{1,2, \cdots, T\}$

$I$ : Set of assets $\{1,2, \cdots, n\}$

$x_{i}^{t}$ : Output of asset $i$ at time $t, i \in I, t \in \mathfrak{T}$

$d_{i}^{t}$ : Demand generated in asset $i$ at time $t, i \in I, t \in \mathfrak{T}$

$c_{i}^{t}$ : Production cost in asset $i$ at time $t, i \in I, t \in \mathfrak{T}$

$p_{i}^{t}$ : Price of asset $i$ at time $t, i \in I, t \in \mathfrak{T}$

$P_{i}^{t}$ : Maximum productive capacity of asset $i$ at time $t, i \in I, t \in \mathfrak{T}$

$v_{i}^{t}$ : Inventory of asset $i$ at time $t, i \in I, t \in \mathfrak{T}$

$m_{i}^{t}$ : Unit inventory cost in asset $i$ at time $t, i \in I, t \in \mathfrak{T}$

$Q_{i}$ : Maximum productive capacity of asset $i, i \in I$

$C$ : Maximum inventory capacity.

For this problem, the aim is to maximum reward and the deterministic optimization model presented in [18] is as follows:

$$
\begin{array}{ll}
\max _{x, v} & \sum_{i \in I} \sum_{t \in \mathfrak{T}} p_{i}^{t} d_{i}^{t}-\sum_{i \in I} \sum_{t \in \mathfrak{T}} c_{i}^{t} x_{i}^{t}-\sum_{i \in I} \sum_{t \in \mathfrak{T}} m_{i}^{t} v_{i}^{t} \\
\text { s.t. } & v_{i}^{t+1} \leq v_{i}^{t}+x_{i}^{t}-d_{i}^{t}, \forall i \in I, t \in\{1, \cdots, T-1\}, \\
& 0 \leq x_{i}^{t} \leq P_{i}^{t}, \forall i \in I, t \in \mathfrak{T}, \\
& 0 \leq \sum_{t \in \mathfrak{T}} x_{i}^{t} \leq Q_{i}, \forall i \in I, t \in \mathfrak{T}, \\
& v_{i}^{t} \geq 0, \forall i \in I, t \in \mathfrak{T}, \\
& \sum_{i \in I} v_{i}^{t} \leq C, \forall t \in \mathfrak{T}, \\
& v_{i}^{1}=0, \forall i \in I .
\end{array}
$$

Now we consider robust counterpart in terms of uncertain demand $d_{i}^{t}$ like in [22]. That is, it is assumed that demand $d_{i}^{t}$ is unknown and belongs to a prescribed polyhedral uncertainty set:

$$
d_{i}^{t} \in U=\left\{d_{i}^{t}:(1-\theta) \hat{d}_{i}^{t} \leq d_{i}^{t} \leq(1+\theta) \hat{d}_{i}^{t}, \sum_{t \in \mathfrak{T}} d_{i}^{t} \leq D_{i}, \forall i \in I, t \in \mathfrak{T}\right\},
$$

where $\theta$ is uncertainty level, $\hat{d}_{i}^{t}$ is nominal demand in asset $i$ during time interval $t$ and $D_{i}$ is an upper bound for demand in asset $i$. The adjustable control variables $x_{i}^{t}$ and state variables $v_{i}^{t}$ can be represented as the following affine functions of the previously observed demand, i.e., 


$$
\begin{gathered}
x_{i}^{t}=\eta_{i t}^{\prime}+\sum_{s \in I} \sum_{\tau \in I_{t}} \eta_{i t}^{s \tau} d_{s}^{\tau}, \\
v_{i}^{t}=\pi_{i t}^{\prime}+\sum_{s \in I} \sum_{\tau \in I_{t}} \pi_{i t}^{s \tau} d_{s}^{\tau},
\end{gathered}
$$

where $\eta_{i t}^{\prime}, \eta_{i t}^{s \tau}, \pi_{i t}^{\prime}$ and $\pi_{i t}^{s \tau}$ are non-adjustable variables and $I_{t}=\{0,1, \cdots, t-1\}$.

\subsection{The Proposed Model for this Problem}

By substituting the state and control variables, we obtain the new affinely adjustable robust counterpart of model in Equation (8) with the shared uncertain $d_{i}^{t}$ as follows:

$$
\max _{\eta, \eta^{\prime}, \pi, \pi^{\prime}}\left\{\begin{array}{cl}
\min _{d \in U} \quad \sum_{i \in I} \sum_{t \in \mathfrak{T}} p_{i}^{t} d_{i}^{t}-\sum_{i \in I} \sum_{t \in \mathfrak{T}} c_{i}^{t}\left(\eta_{i t}^{\prime}+\sum_{s \in I} \sum_{\tau \in I_{t}} \eta_{i t}^{s \tau} d_{s}^{\tau}\right) \\
-\sum_{i \in I} \sum_{t \in \mathfrak{T}} m_{i}^{t}\left(\pi_{i t}^{\prime}+\sum_{s \in I} \sum_{\tau \in I_{t}} \pi_{i t}^{s \tau} d_{s}^{\tau}\right) \\
\text { s.t. } \quad \pi_{i t+1}^{\prime}+\sum_{s \in I} \sum_{\tau \in I_{t}} \pi_{i t+1}^{s \tau} d_{s}^{\tau} \\
\leq\left(\pi_{i t}^{\prime}+\sum_{s \in I} \sum_{\tau \in I_{t}} \pi_{i t}^{s \tau} d_{s}^{\tau}\right)+\left(\eta_{i t}^{\prime}+\sum_{s \in I} \sum_{\tau \in I_{t}} \eta_{i t}^{s \tau} d_{s}^{\tau}\right)-d_{i}^{t}, \\
\forall i \in I, t \in\{1, \cdots, T-1\}, \\
0 \leq \eta_{i t}^{\prime}+\sum_{s \in I} \sum_{\tau \in I_{t}} \eta_{i t}^{s \tau} d_{s}^{\tau} \leq P_{i}^{t}, \forall i \in I, t \in \mathfrak{T}, \\
0 \leq \sum_{t \in \mathfrak{T}}\left(\eta_{i t}^{\prime}+\sum_{s \in I} \sum_{\tau \in I_{t}} \eta_{i t}^{s \tau} d_{s}^{\tau}\right) \leq Q_{i}, \forall i \in I, t \in \mathfrak{T}, \\
\pi_{i t}^{\prime}+\sum_{s \in I} \sum_{\tau \in I_{t}} \pi_{i t}^{s \tau} d_{s}^{\tau} \geq 0, \forall i \in I, t \in \mathfrak{T}, \\
\text { s.t. } \quad \sum_{i \in I}^{\prime} \pi_{i t}^{\prime}+\sum_{s \in I} \sum_{\tau \in I_{t}} \pi_{i t}^{s \tau} d_{s}^{\tau} \leq C, \forall t \in \mathfrak{T} .
\end{array}\right\}
$$

In this model, the objective represents the worst-case reward under the variable $d$ satisfying all related constraints, i.e., the inner problem in Equation (9). We denote all inequality constraints in Equation (9) as $S\left(\eta, \eta^{\prime}, \pi, \pi^{\prime}, d\right) \geq 0$ for short. We can utilize duality theory to deal with this complicated max-min problem. We demonstrate this approach in the following theorem.

Theorem 2. Suppose that, for all fixed $\eta, \eta^{\prime}, \pi$, and $\pi^{\prime}$ concerned in the outer maximizing problem of Equation (9), there exists a $\hat{d} \in U$, such that

$$
S\left(\eta, \eta^{\prime}, \pi, \pi^{\prime}, \hat{d}\right) \geq 0
$$

Then the model in Equation (9) is equivalent to the following nonlinear programming problem: 


$$
\begin{aligned}
& \max _{\lambda, \eta, \eta^{\prime}, \pi, \pi^{\prime}} \sum_{i \in I} \sum_{t \in \mathfrak{T}}\left[-c_{i}^{t} \eta_{i t}^{\prime}-m_{i}^{t} \pi_{i t}^{\prime}-\lambda_{i t}^{b} \eta_{i t}^{\prime}+\lambda_{i t}^{c}\left(\eta_{i t}^{\prime}-P_{i}^{t}\right)+\lambda_{i}^{d} \eta_{i}^{\prime}\right. \\
& \left.-\lambda_{i t}^{e} \pi_{i t}^{\prime}+\lambda_{t}^{f} \pi_{i t}^{\prime}+(1-\theta) \lambda_{i t}^{g a} \hat{d}_{i}^{t}-(1+\theta) \lambda_{i t}^{g b} \hat{d}_{i}^{t}\right] \\
& +\sum_{i \in I} \sum_{t \in\{1, \cdots, T-1\}} \lambda_{i t}^{a}\left(\pi_{i t+1}^{\prime}-\pi_{i t}^{\prime}-\eta_{i t}^{\prime}\right) \\
& -\sum_{i \in I}\left(\lambda_{i}^{d} Q_{i}+\lambda_{i}^{g c} D_{i}\right)-\sum_{t \in \mathfrak{T}} \lambda_{t}^{f} C \\
& \text { s.t. } \quad \sum_{i \in I} \sum_{t \in\{\tau+1, \cdots, T-1\}} \lambda_{i t}^{a}\left(\pi_{i t}^{s \tau}+\eta_{i t}^{s \tau}-\pi_{i t+1}^{s \tau}\right)-\lambda_{s \tau}^{a}+\lambda_{s \tau}^{g a}-\lambda_{s \tau}^{g b}-\lambda_{s}^{g c} \\
& +\sum_{i \in I} \sum_{t \in\{\tau+1, \cdots, T\}}\left[\left(\lambda_{i t}^{b}-\lambda_{i t}^{c}-\lambda_{i}^{d}\right) \eta_{i t}^{s \tau}+\left(\lambda_{i t}^{e}-\lambda_{t}^{f}\right) \pi_{i t}^{s \tau}\right] \\
& +\sum_{i \in I} \lambda_{i \tau}^{a} \pi_{i \tau+1}^{s \tau}=P_{s}^{\tau}-\sum_{i \in I} \sum_{t \in\{\tau+1, \cdots, T\}}\left(c_{i}^{t} \eta_{i t}^{s \tau}+m_{i}^{t} \pi_{i t}^{s \tau}\right), \\
& \forall s \in I, \tau \in\{1, \cdots, T-2\} \\
& \sum_{i \in I} \pi_{i T}^{s \tau}\left(-\lambda_{i T-1}^{a}+\lambda_{i T}^{e}-\lambda_{T}^{f}\right)+\sum_{i \in I} \eta_{i T}^{s \tau}\left(\lambda_{i T}^{b}-\lambda_{i T}^{c}-\lambda_{i}^{d}\right) \\
& -\lambda_{s \tau}^{a}+\lambda_{s \tau}^{g a}-\lambda_{s \tau}^{g b}-\lambda_{s}^{g c}=P_{s}^{\tau}-\sum_{i \in I}\left(c_{i}^{T} \eta_{i T}^{s \tau}+m_{i}^{T} \pi_{i T}^{s \tau}\right), \\
& \forall s \in I, \tau=T-1 \text {, } \\
& P_{s}^{T}=\lambda_{s T}^{g a}-\lambda_{s T}^{g b}-\lambda_{s}^{g c}, \forall s \in I, \\
& S\left(\eta, \eta^{\prime}, \pi, \pi^{\prime}, \hat{d}\right) \geq 0, \lambda \geq 0, \pi_{i 1}^{\prime}=0, \forall i \in I \text {. }
\end{aligned}
$$

Proof. By the assumption, there must exists a $\hat{d}$ such that $S\left(\eta, \eta^{\prime}, \pi, \pi^{\prime}, \hat{d}\right) \geq 0$. Therefore, the inner minimization over $d$ in Equation (9) is feasible. In addition, it is easy to verify the inner minimization in the model in Equation (9) is also bounded below. For each fixed $\eta, \eta^{\prime}, \pi$ and $\pi^{\prime}$, we obtain that the strong duality property holds for the inner minimization and then the inner minimization problem can be equivalent to a dual problem (i.e., a maximization problem) by the linear duality theorem. Finally, combining the dual problem with the outer maximization in Equation (9), we can obtain the model in Equation (10). Here we omit detailed derivation since it is an example of the duality theorem in LP.

Even though we reformulate the affinely adjustable robust optimization as a nonconvex programming problem, many commercial nonlinear optimization solvers can be utilized to solve the problem in Equation (10). Besides, we should note that the model in Equation (10) not only involves less variables but also has more practical significance compared with the model in Equation (12) in [22] and the model in Equation (20) in [18].

\section{Evaluation for the Proposed Model}

In this section, we apply the proposed robust model of Equation (9) and its equivalent dual form in Equation (10) under the uncertain set $U$ to solve a real multi-stage logistics production and inventory process problem to show the superiority of our robust model. Numerical results will show that our proposed model can give an effective strategy so that investors would receive preferable rewards. More details is as follows.

\subsection{Experimental Details}

All numerical experiments were run under Matlab version R2015b on a Thinkpad laptop computer with an Intel Core i5 processor at $2.5 \mathrm{GHz}$ with 4 GB RAM. Since the optimization model in Equation (10) is nonconvex and nonlinear, we use the commercial nonlinear optimization solver KNITRO (Available at https:/ /www.artelys.com/en/optimization-tools/knitro) to solve our model.

According to [18], denote logistics products $n=10$, production planning period $T=10$, nominal demand $\hat{d}_{i}^{t}=100(1+0.1)^{t}$, and upper bound for demand in asset $i$ as $D_{i}=2 \sum d_{i}^{t}$, and

$$
p_{i}^{t}=100(1+0.05)^{t}, c_{i}^{t}=30(1+0.05)^{t}, m_{i}^{t}=2(1+0.05)^{t},
$$




$$
P_{i}^{t}=300(1+0.05)^{t}, q_{i}=\sum P_{i}^{t}, C=\sum q_{i} .
$$

Next, we compare our model in Equation (10) with the AARC1 model in [18]. The Affinely Adjustable Robust Counterpart (AARC) is first proposed by Ben-Tal et al. in [24]. AARC model restricts the adjustable variables to be affine functions of the uncertain data and converts the NP-hard Adjustable Robust Counterpart (ARC) problem to be a semi-infinite conic programming. Then it can be reformulated as a computationally tractable problem (typically an LP or a semidefinite problem) which is denoted by AARC1 model in [18]. Since the AARC1 model for this multi-stage logistics production and inventory process problem is an LP, we use the commercial solver CPLEX to solve it. Here we test the two models against different levels of uncertainty, specifically, varying $\theta$ from 0 to 1 with increment 0.1 .

\subsection{Results and Evaluations}

We output the optimal objective of the two models vs different uncertainty level $\theta$ in Figure 1. That is, Figure 1 shows the maximal rewards of two models with different uncertainty levels, respectively. We also plot the histogram of total profit of the 10 periods to comparison more clearly in Figure 2.

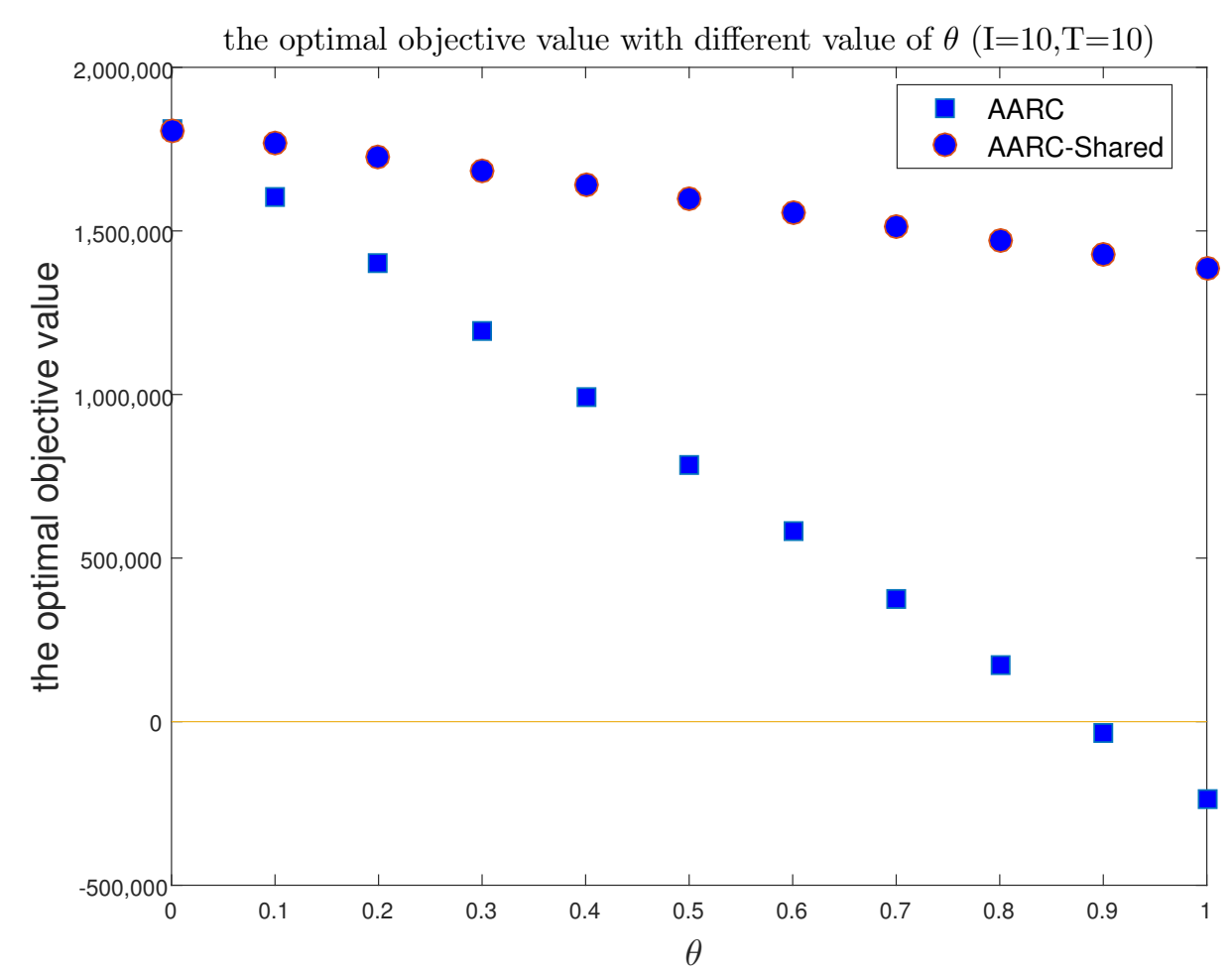

Figure 1. The comparison between our model (Affinely Adjustable Robust Counterpart (AARC)-Shared) and AARC model in [18]. 


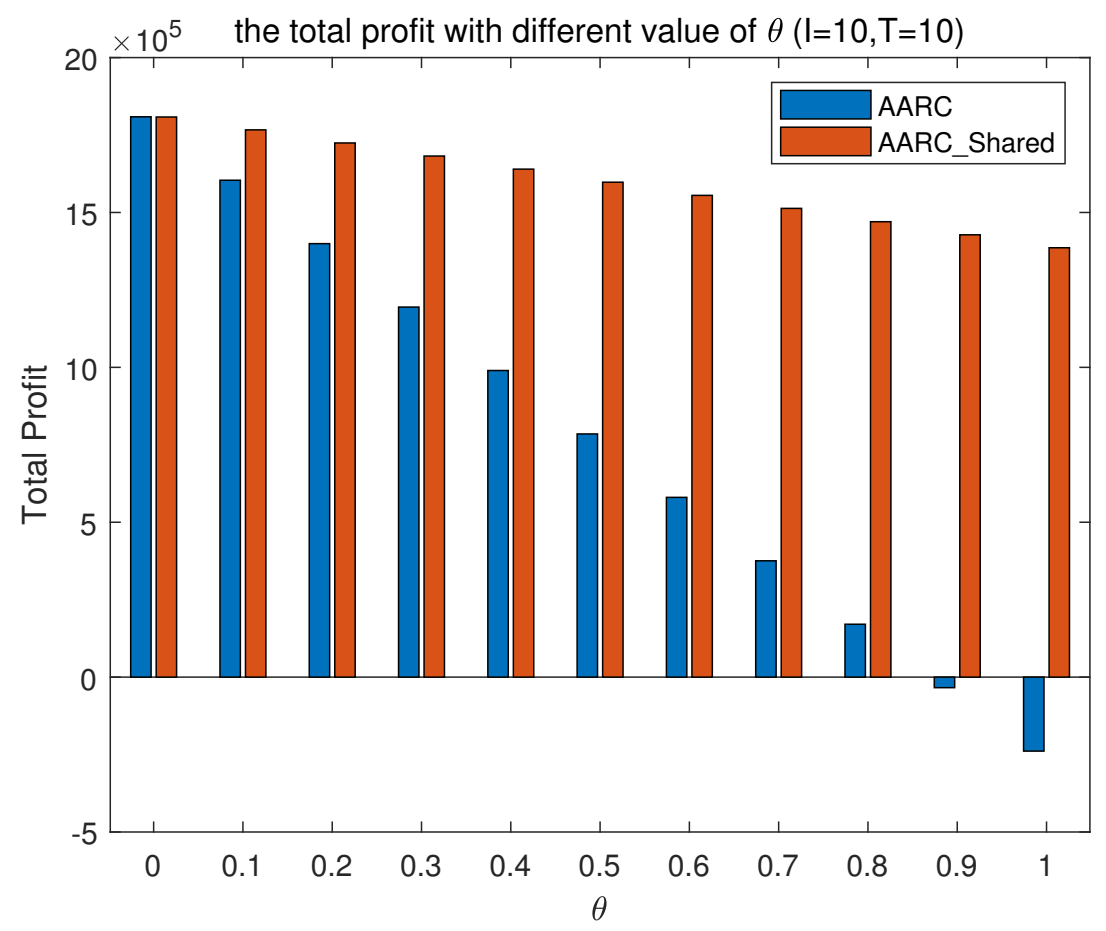

Figure 2. The total profit obtained by two models.

We can see that the maximal rewards decrease along with increasing of the level of uncertainty $\theta$ for both models. Here it should be clarified that it is a deterministic multi-stage logistics production and inventory process problem when $\theta=0$. Obviously, the optimal value of reward in the deterministic case is lager than ones in any other uncertain cases. It exactly illustrates the meaning of robust as we expect. This is easily understandable because the optimal values of both models can be interpreted as the optimistic estimate of total reward in the worst case, which can be lower and lower as the level of uncertainty increases (i.e., the robust feasible region gets larger and larger). This can be interpreted by Figure 3 which shows the demand $d_{i}^{5}$ (the 5 th period) with different uncertain level $\theta$.

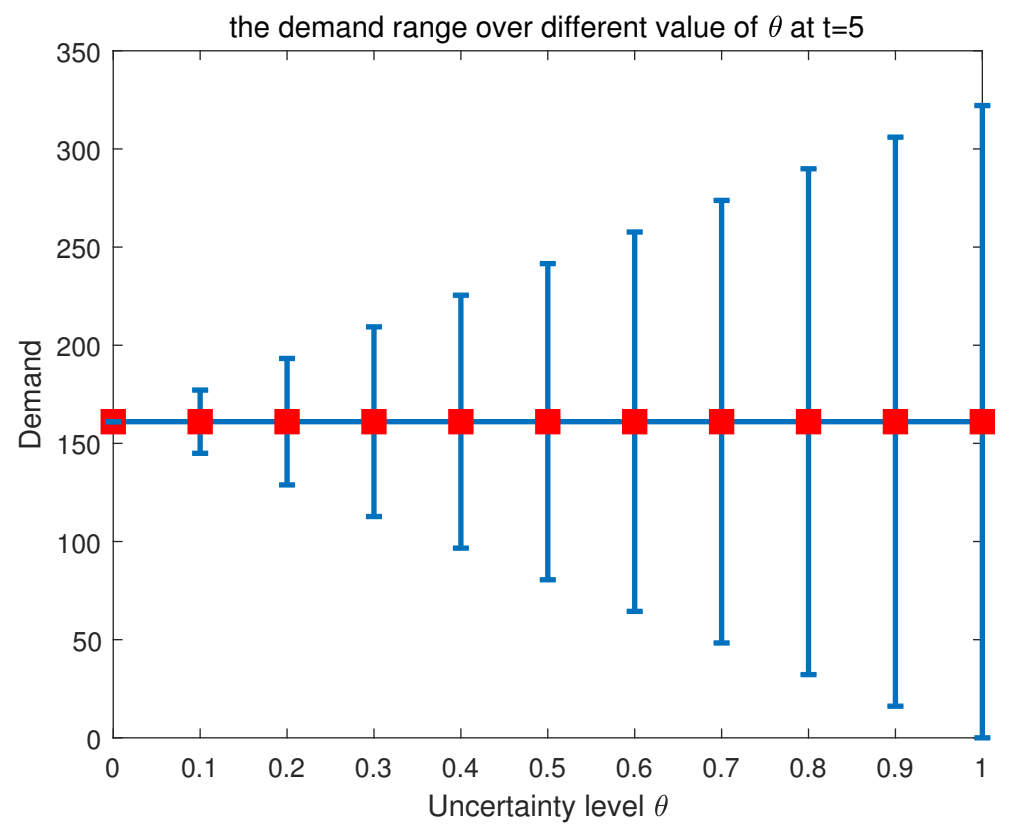

Figure 3. The impact of $\theta$ to uncertain demand (e.g., $t=5$ ). 
However, compared with our model, the optimal values solved by AARC1 decrease dramatically as $\theta$ increasing and specially it can even become negative when $\theta=0.9$ and 1 . This is because AARC1 considers its feasible solutions (for $\eta, \eta^{\prime}, \pi, \pi^{\prime}$ ) satisfying each constraint for all $d_{i}^{t} \in U$. Figure 4 plots the range of $d_{i}^{t}$ in all 10 periods over $\theta=0.9$.

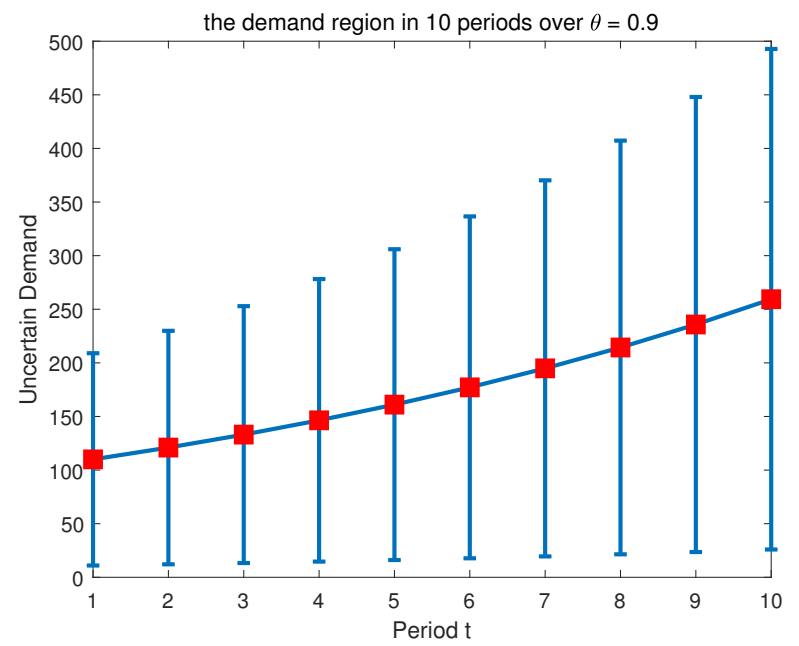

Figure 4. The demand range in all periods over $\theta=0.9$.

Clearly, as $\theta$ increases, the range of $d_{i}^{t}$ enlarges which make the outer feasible region of AARC1 shrink rapidly. On the other hand, no matter how the company arranges the production and inventory planning, the model in [18] tells people that they will run at a loss in the worst cases when $\theta=0.9$ and 1 . This is too conservative to give a production and inventory plan. While, our AARC-Shared model is less conservative to some extent and can give a valid strategy in the production and inventory planning under a larger uncertainty level than the AARC1 model.

\section{Conclusions}

In this paper, the robust optimization model with shared uncertain parameters is proposed. Unlike existing robust models, we only consider the worst case of the objective under the constraints sharing the same uncertain variable and regard it as in the inner problem of entire model. In terms of the duality theorem, the proposed robust model can be reformulated to a nonconvex optimization problem step by step.

Compared with existing robust optimization models in [14,16-18,22-24] and so on, the proposed model has two advantages:

1. Normally, the same variable in one problem indicates the same meaning. So the uncertain situation of the same variable in robust optimization should be the same in one event. In view of this point, our model focuses on the optimization problems under the same uncertain situation shared in both the objective and constraints, and is more practical and realistic.

2. Our model is less conservative and can provide more flexibility in regards to practical problems. When people set more conditions and constraints to models in terms of personal preference, our new robust model can have a greater chance to solve the problems than the original robust models, since they are more conservative with their feasible region. Therefore, the robust optimization model with shared uncertain parameters can be an advisable alternative for investors and the investors can make a decision more actively, but not too conservative by our model.

Author Contributions: Conceptualization, L.X. and Y.Z.; methodology, B.Y.; software, L.X.; validation, L.X., Y.Z., and B.Y.; formal analysis, L.X. and Y.Z.; writing-original draft preparation, Y.Z. and L.X; visualization, L.X.; supervision, B.Y. All authors have read and agreed to the published version of the manuscript. 
Funding: This research was funded by Natural Science Foundation of Liaoning Province of China (NO. 2019-BS-013), National Natural Science Foundation of China (NO. 11971092) and the Fundamental Research Funds for the Central Universities (No. DLMU3132019323).

Acknowledgments: We will thank the anonymous reviewers for taking time off their busy schedule to review this paper.

Conflicts of Interest: The authors declare no conflict of interest.

\section{References}

1. Bertsimas, D.; Brown, D.B.; Caramanis, C. Theory and applications of robust optimization. SIAM Rev. 2011, 53, 464-501. [CrossRef]

2. Bertsimas, D.; Brown, D.B. Constructing uncertainty sets for robust linear optimization. Oper. Res. 2009, 57, 1483-1495. [CrossRef]

3. Ben-Tal, A.; Bertsimas, D.; Brown, D.B. A soft robust model for optimization under ambiguity. Oper. Res. 2010, 58, 1220-1234. [CrossRef]

4. Bertsimas, D.; Goyal, V. On the power of robust solutions in two-stage stochastic and adaptive optimization problems. Math. Oper. Res. 2010, 35, 284-305. [CrossRef]

5. Delage, E.; Ye, Y. Distributionally Robust Optimization Under Moment Uncertainty with Application to Data-Driven Problems. Oper. Res. 2010, 58, 595-612. [CrossRef]

6. Luo, G.M.; Li, D.H. Robust optimization equilibrium with deviation measures. Pac. J. Optim. 2009, 5, 427-442.

7. Xu, H.; Caramanis, C.; Mannor, S. Robust regression and Lasso. IEEE Trans. Inform. Theory 2010, 56, 3561-3574. [CrossRef]

8. Xu, H.; Caramanis, C.; Mannor, S. A distributional interpretation of robust optimization. Math. Oper. Res. 2012, 37, 95-110. [CrossRef]

9. Sun, H.; Xu, H. Convergence analysis for distributionally robust optimization and equilibrium problems. Math. Oper. Res. 2016, 41,377-401. [CrossRef]

10. Tong, X.; Sun, H.; Luo, X.; Zheng, Q. Distributionally robust chance constrained optimization for economic dispatch in renewable energy integrated systems. J. Glob. Optim. 2018, 70, 131-158. [CrossRef]

11. Xu, H.; Liu, Y.; Sun, H. Distributionally robust optimization with matrix moment constraints: Lagrange duality and cutting plane methods. Math. Program. 2018, 169, 489-529. [CrossRef]

12. Chen, X.; Sun, H.; Xu, H. Discrete approximation of two-stage stochastic and distributionally robust linear complementarity problems. Math. Program. 2019, 177, 255-289. [CrossRef]

13. Atta Mills, E.; Yu, B.; Zeng, K. Satisfying Bank Capital Requirements: A Robustness Approach in a Modified Roy Safety-First Framework. Mathematics 2019, 7, 593. [CrossRef]

14. Goldfarb, D.; Iyengar, G. Robust portfolio selection problems. Math. Oper. Res. 2003, 28, 1-38. [CrossRef]

15. Tütüncü, R.H.; Koenig, M. Robust asset allocation. Ann. Oper. Res. 2004, 132, 157-187. [CrossRef]

16. Zhu, S.S.; Fukushima, M. Worst-case conditional value-at-risk with application to robust portfolio management. Oper. Res. 2009, 57, 1155-1168. [CrossRef]

17. Tong, X.J.; Wu, F. Robust reward-risk ratio optimization with application in allocation of generation asset. Optimization 2012, 1-19. [CrossRef]

18. Yao, C. Issues in Multi-stage Logistics Production and Inventory Process Based on Adjustable Robust Optimization. Logist. Technol. 2014, 33, 231-233.

19. Zhou, Y.; Yang, L.; Xu, L.; Yu, B. Inseparable robust reward-risk optimization models with distribution uncertainty. Jpn. J. Ind. Appl. Math. 2016, 33, 767-780. [CrossRef]

20. Tong, X.J.; Wu, F.; Qi, L.Q. Worst-case CVaR based portfolio optimization models with applications to scenario planning. Optim. Methods Softw. 2009, 24, 933-958. [CrossRef]

21. Xu, L.; Yu, B.; Liu, W. The Distributionally Robust Optimization Reformulation for Stochastic Complementarity Problems. Abstr. Appl. Anal. 2014, 469587, 1-7. [CrossRef]

22. Ben-Tal, A.; Chung, B.D.; Mandala, S.R.; Yao, T. Robust optimization for emergency logistic planning: Risk mitigation in hunmanitarian relief supply chains. Transp. Res. Part B 2009, 45, 177-189. [CrossRef]

23. Ben-Tal, A.; Golany, B.; Shtern, S. Robust Multi Echelon Multi Period Inventory Control. Eur. J. Oper. Res. 2009, 199, 922-935. 
24. Ben-Tal, A.; Goryashko, A.; Guslitzer, E.; Nemirovski, A. Adjustable robust solutions of uncertain linear programs. Math. Program. 2004, 99, 351-376. [CrossRef]

(C) 2020 by the authors. Licensee MDPI, Basel, Switzerland. This article is an open access article distributed under the terms and conditions of the Creative Commons Attribution (CC BY) license (http://creativecommons.org/licenses/by/4.0/). 